\title{
Modified Watershed Algorithm for Segmentation of 2D Images
}

\author{
Malik Sikandar Hayat Khiyal, Aihab Khan, and Amna Bibi \\ Computer Sciences Dept., Fatima Jinnah Women University, \\ Rawalpindi, Pakistan
}

\author{
m.sikandarhayat@yahoo.com; aihabkhan@yahoo.com; \\ gul star3@yahoo.com
}

\begin{abstract}
With the repaid advancement of computer technology, the use of computer-based technologies is increasing in different fie lds of life. Image segmentation is an important problem in different fields of image processing and computer vision. Image segmentation is the process of dividing images according to its characteristic e.g., color and objects present in the images. Different methods are presented for image segmentation. The focus of this study is the watershed segmentation. The tool used in this study is MATLAB. Good result of watershed segmentation entirely relay on the image contrast. Image contrast may be degraded during image acquisition. Watershed algorithm can generate over segmentation or under segmentation on badly contrast images. In order to reduce these deficiencies of watershed algorithm a preprocessing step using Random Walk method is performed on input images. Random Walk method is a probabilistic approach, which improves the image contrast in the way image is degraded.
\end{abstract}

Keywords: image processing, segmentation, watershed transformation.

\section{Introduction}

In image processing, segmentation is a basic problem in different fields for example, pattern recognition, scene analysis and image analysis. Image segmentation is the process of dividing images into regions according to its characteristic e.g., color and objects present in the images. These regions are sets of pixels and have some meaningful information about object. The result of image segmentation is in the form of images that are more meaningful, easier to understand and easier to analyze. In order to locate objects and boundaries in images feature extraction of object shape, optical density, and texture, surface visualization, image registration and compression image segmentation is used. Correct segmented results are very useful for the analysis, predication and diagnoses (Segmentation (image processing), n.d.).

Material published as part of this publication, either on-line or in print, is copy righted by the Informing Science Institute. Permission to make digital or paper copy of part or all of these works for personal or classroom use is granted without fee provided that the copies are not made or distributed for profit or commercial advantage AND that copies 1) bear this notice in full and 2) give the full citation on the first page. It is permissible to abstract these works so long as cred it is given. To copy in all other cases or to republish or to post on a server or to redistribute to lists requires specific permission and payment of a fee. Contact Publisher@InformingScience.org to request redistribution permission.
Watershed segmentation is a morphological based method of image segmentation. The gradient magnitude of an image is considered as a topographic surface for the watershed transformation. Watershed lines can be found by different ways. The complete division of the image through watershed transformation relies mostly on a good estimation of image gradients. The result of the watershed transform is de- 
graded by the background noise and produces the over-segmentation. Also, under segmentation is produced by low-contrast edges generate small magnitude gradients, causing distinct regions to be erroneously merged.

In order to reduce the deficiencies of watershed, many preprocessing techniques are proposed by the different researchers for example Jung and Scharcanski (2005) presents a robust watershed segmentation using wavelets where wavelets technique is used to denoise the image. Bieniek and Moga (2000) present an efficient watershed algorithm based on connected components. Hamarneh and $\mathrm{Li}$ (2009) have proposed a method of watershed segmentation using prior shape and appearance knowledge to improve the segmentation results etc. But most of the techniques previously proposed consider the over segmentation problems and focus on the denoising of the image. The image low contrast and under segmentation problem is not yet addressed by most of the researchers.

The proposed technique focuses on the solution of under segmentation problem of low contrast images by applying preprocessing on the input image. The technique for preprocessing on the images is Random Walk (Erikson, 2005). It is probabilistic approach used to enhance the image contrast in the way image is degraded.

The division of this paper is as follows, in section 2 some related work is given which describes the previous research about the remedy of watershed issues. In section 3, some basic information about watershed and random walk is given. In section 4, the proposed technique is discussed. In section 5, results are given. In section 6, the conclusion and future plane is given.

\section{Related Work}

Erikson (2005) proposes two different techniques of performing preprocessing of an image to improve segmentation results. The methods use the grey level thickness of the objects, in order to find the resulting image. The first method proposed by the author is RW, uses the random walk of a particle to a random position, the position is defined in the neighborhood of the particle. The resulting image through this method holds the number of times the particle visits a pixel. The second method is Iterative Procedure (IP) scans the image iteratively and calculates the expected value of the same number, instead of randomization to find the number of visits. The methods proposed in this paper are independent of the segmentation method and can therefore be used as a preprocessing step for other segmentation methods as well.

Beucher (1991) proposed a method for image segmentation based on the mathematical morphology. The process of image segmentation is divides into two approaches, boundary based and region based. Watershed segmentation is a region based approach and uses to detect the pixel and region similarities. Basic tools for the watershed transformation are given and watershed transformation is applied on the gray tone images by using flooding process. The problem of over segmentation is remedied by using marker controlled watershed segmentation. Another approach, called hierarchical segmentation, is proposed. This approach is particularly effic ient for defining levels of segmentation starting from a graph representation of the images based on the mosaic image transform.

Hsieh, Han, Wu, Chuangc, and Fana (2006) proposed a novel approach for small object detection by using watershed-based transformation. In this paper, the small moving objects are detected from the image. In order to improve the detection results from the previous techniques a noise removal technique is first applied to the image which removes the noise from the image and improve the image quality. The proposed detection system includes two main modules, first one is region of interest (ROI) locating and the other is contour extraction. After noise removal accurate ROI can be located. In contour extraction process, a rough candidate object in the images can be detected by applying some differencing technique on two contagious image frames. In this paper, 
in order to find the best results for contour extraction, a watershed-based technique along with the region matching technique is applied.

Hamarneh and $\mathrm{Li}$ (2007) propose a method for image segmentation consist of watershed segmentation using prior shape and appearance knowledge. Watershed segmentation is a common technique for image segmentation but has problems of over segmentation and sensitivity to noise. The proposed method has two stages, first is training stage and the other is segmentation stage. In training stage, a prior shape and appearance knowledge model is developed by using 'shape histogram' and image intensity statistics. The segmentation stage is an automatic iterative procedure and consists of four steps: classical watershed transformation, improved k-means clustering, shape alignment, and refinement. The issues of watershed are remedied by this method, as over segmentation problem is handled by clustering and noise effect can be removed by mean intensity of each segment. The limitation of k-mean clustering algorithm affects the proposed methods result and a failure case is reported.

Other researchers also proposed different method to remedy the problem of watershed. Li, Elmoataz, Fadili, and Ruan, S. (2003) proposed an improved image segmentation approach based on level set and mathematical morphology. The gradient magnitude of the smoothed image is input to the watershed transformation, the result of watershed is used for rough approximation of the desired contour in the image, and guide for the initial location of the seed points used in the following level set method. This method combines the advantages of both the methods and finds best contoured.

\section{Basic Theory}

This section gives some basic information about Watershed segmentation and Random Walk method.

\section{Watershed Transformation}

Watershed transformation is a morphological based tool for image segmentation. In grey scale mathematical morphology the watershed transformation for image segmentation is originally proposed by Digabel and Lantuejoul (1977) and later improved by Li et. al. (2003). The watershed transform can be classified as a region-based segmentation approach.

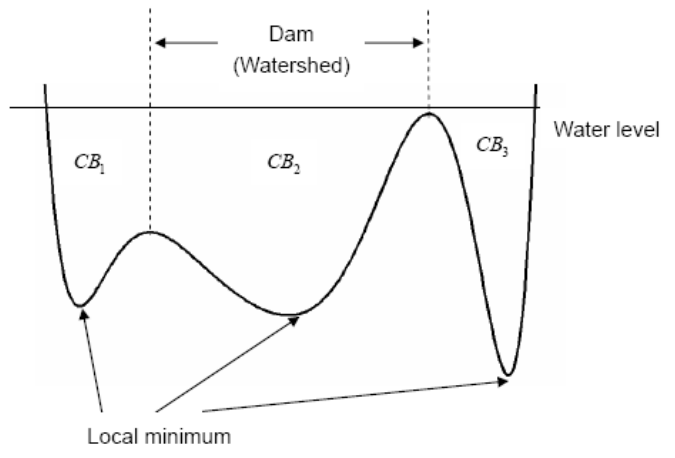

Figure 1: Illustration of immersion process of watershed transforms. (CB is for catchment basins)

The idea of watershed can be view as a landscape immersed in a lake; catchment basins will be filled up with water starting at each local minimum. Dams must be built where the water coming from different catchment basins may be meeting in order to avoid the merging of catchment basins. The water shed lines are defined by the catchment basins divided by the dam at the highest level where the water can reach in the landscape. As a result, watershed lines can separate indi- 
vidual catchment basins in the landscape. The idea is described in Figure 1 which describes the flooding or rain falling process of watershed algor ithm (Hsiesh, 2006).

The process of rain falling is described in Figure 2.

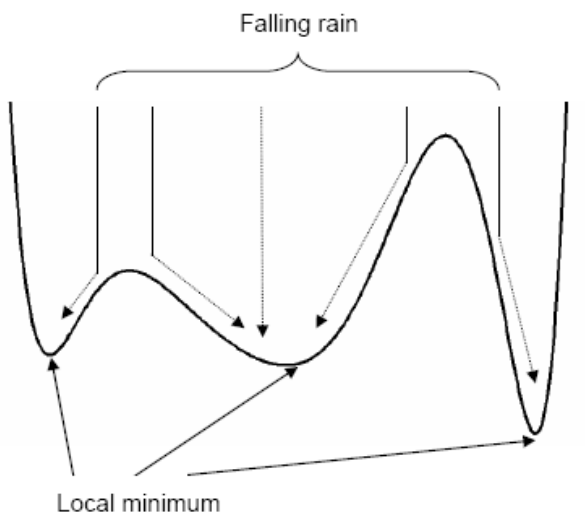

Figure 2: Illustrations of flooding (rain-falling) process of watershed transform.

\section{Random Walk}

Random Walk is a process of image enhancement and use to enhance the image in the image is degraded. This method is based on a random walk of a particle. In this method a seed point or starting point must be found from where the particle can start its move. Where the seed point is located is irrelevant. All object pixels will be visited anyway. The resultant image through this method holds the number of times the particle is visiting a pixel (Erikson, 2005).

The move of the particle is start from the seed point and the partic le is jumped to a random position in its neighbor based on the condition that the random gray level is less than the gray level of the randomized position. Erikson (2005) proposed that if a a particle from a seed point $\mathrm{p}$ wants to jump to a random position q. it must satisfied the below condition

if $\mathrm{z}_{1}<\mathrm{f}\left(\mathrm{x}_{1}, \mathrm{y}_{1}\right)$ where $\mathrm{z}_{1}$ is the uniform random number. And $\mathrm{f}\left(\mathrm{x}_{1}, \mathrm{y}_{1}\right)$ is random position in the image.

Otherwise if $z_{1} \geq f\left(x_{1}, y_{1}\right)$ makes a new randomization until a move can be made. If the location of the particle is $p$, the probability to go from pixel $p$ to pixel $q$ is

$$
\operatorname{Pr}[p \rightarrow q]=\frac{f(q)}{\sum_{i \in N_{p}} f(i)}
$$

The numbers of iterations are dependent on the size of image. A rule of thumb is used, which is $\mathrm{N}_{\mathrm{iter}}>100 \times$ number of pixels in object.

\section{Proposed Technique}

The proposed image segmentation system here is able to segment the 2-D images with minimum drawbacks of over segmentation and under segmentation. Before the segmentation algorithm is performed on the acquired images a preprocessing step by using random walk is performed in order to reduce the segmentation draw backs. The steps of the proposed system are shown in Figure 3. 


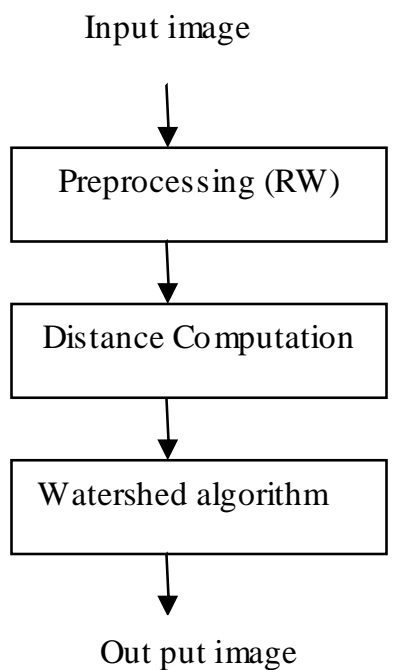

Figure 3: Proposed process for image segmentation

\section{Image Acquisition}

In the step of implementation an image for analys is is acquired. The image can be taken through different sources e.g., camera. Image can have different drawbacks due to the camera fault, poor positioning of the camera, and the object of interest is out of focus. All these drawbacks create difficulty in image analysis especially in image segmentation.

\section{Random Walk}

In order to improve the contrast of the image random walk is applied to the image contrast. This process has the following steps.

Read an image for the system. The input image to the system can be read by using MATLAB built in function.

Convert the image into gray scale. The built in function is used to convert an image into gray scale. Gary scale images has 0 intensity value for black color and 255 for white color and all other pixels have the values between this range dependent on the threshold.

Find the seed point. Seed point is the start point for the particle to make a move. In this research work the seed is found by taking half of the rows and columns.

Calculate threshold. A built in function is used to calculate threshold of the image. This threshold is used to build the neighboring matrix.

Find ne ighboring matrix. In order to find the neighboring matrix, first the number of neighboring position where the particle can jump been found based on the threshold value. Then the neighboring matrix can be found by using this formula

$\mathrm{Np}=\mathrm{I}(\mathrm{x} 0+\mathrm{np}, \mathrm{y} 0+\mathrm{np})$. Where $\mathrm{I}(\mathrm{x} 0, \mathrm{y} 0)$ is the seed point and $\mathrm{np}$ defines the number of positions in the neighborhood.

Make iterations for image enhancement. The number of iteration is dependent on the size of image. A loop is used from 1 to $\mathrm{n}$. 
Find random position. The random position can be found by taking a random pos ition in the neighboring matrix.

Find the random gray level value. In order to find the random gray level value, firstly find the maximum gray level of the image because the random gray level can be in the range of 0 to maximum gray level of the image. Use max function for finding maximum gray level. Then find the value of random gray level by using the formula of uniform random number with in range of 0 to maximum gray level of the image.

Check condition for making move. Check if the random gray level value is less than the gray level value of random position replaces the value with the sum of probability of visits by using the formula. After assigning the new gray level value to the new location pixel, move the seed point to the new location.

After completing the iterations the result of the random walk method is stored in an image.

\section{Watershed Transformation}

After image enhancement by using random walk process. The conventional watershed algorithm is applied on the image. The process of watershed segmentation has the following steps.

Read the RW image.

Convert the image into binary image. MATLAB built in functions are used to convert image into binary images. Binary images can take two possible colors 0 for black and 1 for white color.

Distance transformation. In order to find the object in the image distance transformation is used. Built in function for distance transformation is used which take complement of binary image as an input.

Complement the distance transform and force pixels that don't belong to the objects to be at -Inf.

Apply watershed on the resultant image of previous step and find the watershed ridge lines as 0value pixel are the watershed ridge lines. Then superimpose the watershed ridgelines in the original image.

\section{Results}

The results of the proposed techniques are described in this section.

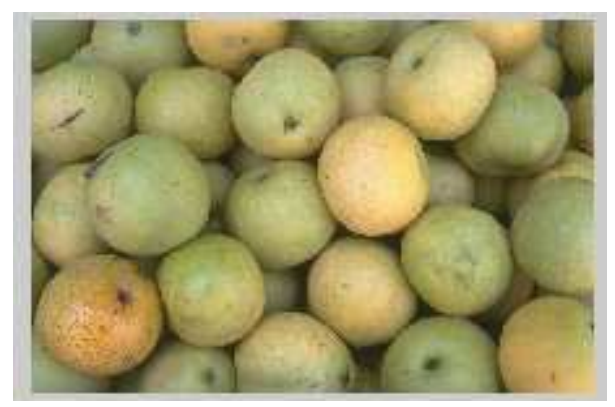

Figure 4: Original image (pears.png)

In Figure 4 the original image is shown. 


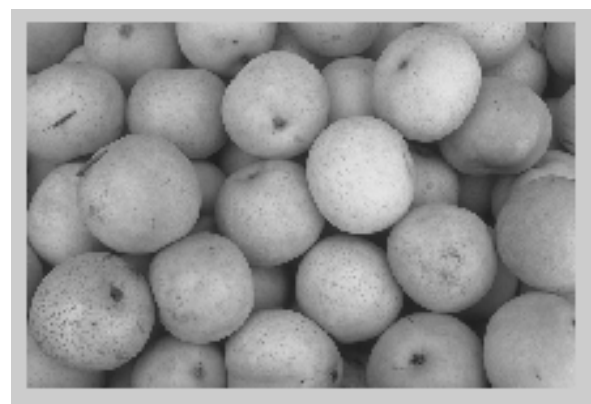

Figure 5: Image after random walk

Figure 5 shows the image after random walk.

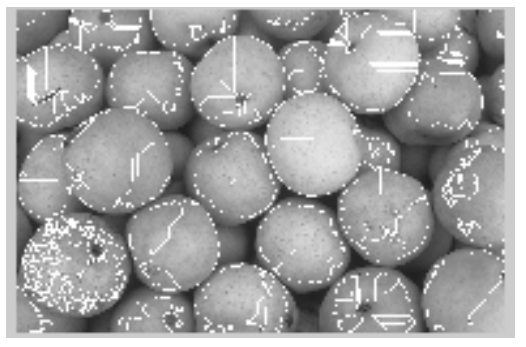

Figure 6: Segmentation results with out using random walk

In Figure 6 the results of watershed segmentation without preprocessing are shown.

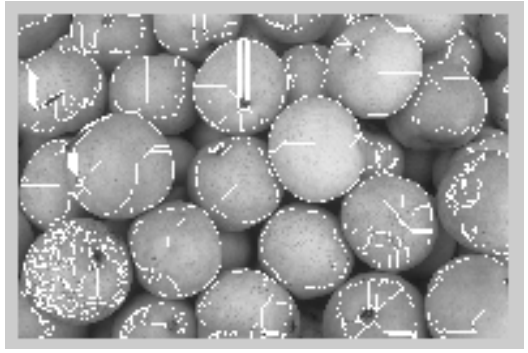

Figure 7: Segmentation results with using random walk

The results of watershed segmentation with the preprocessing using random walk are shown in Figure 7.

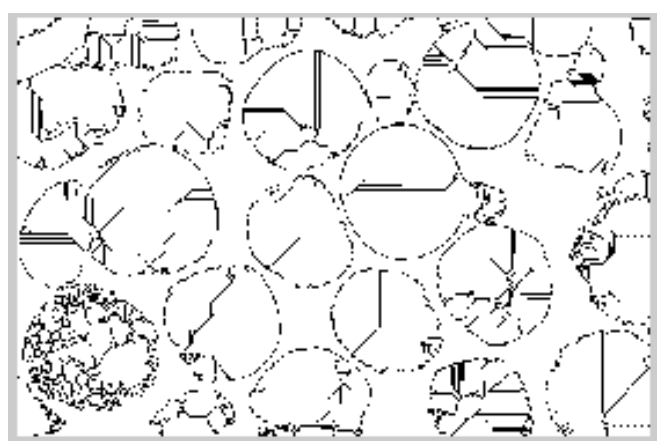

Figure 8: Differences between both segmentation results

The difference between both segmented images is shown in Figure 8.

The results of another image using the same techniques are shown in Figures 9-13. 


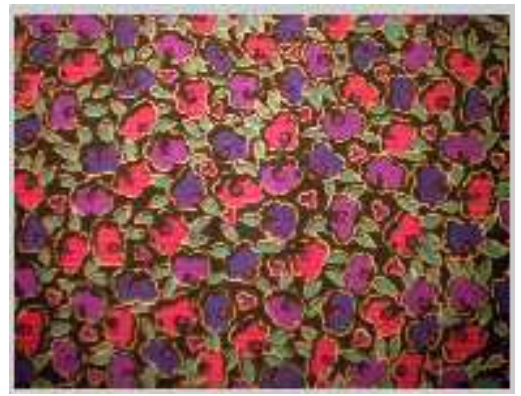

Figure 9: Original image (pears.png)

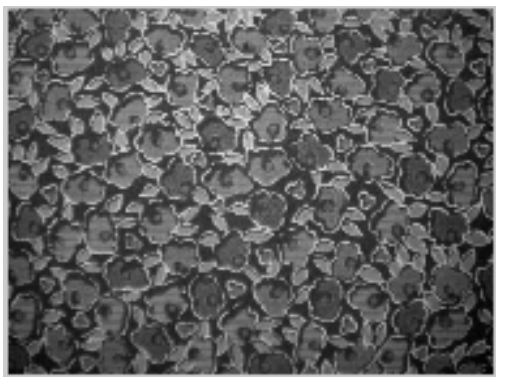

Figure 10: Image after random walk

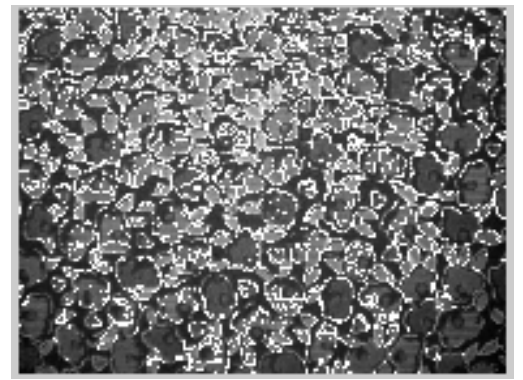

Figure 11: Segmentation results with out using random walk

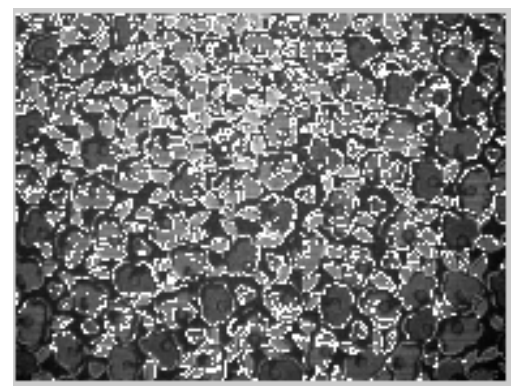

Figure 12: Segmentation results with using random walk 
Khiyal, Khan, \& Bibi

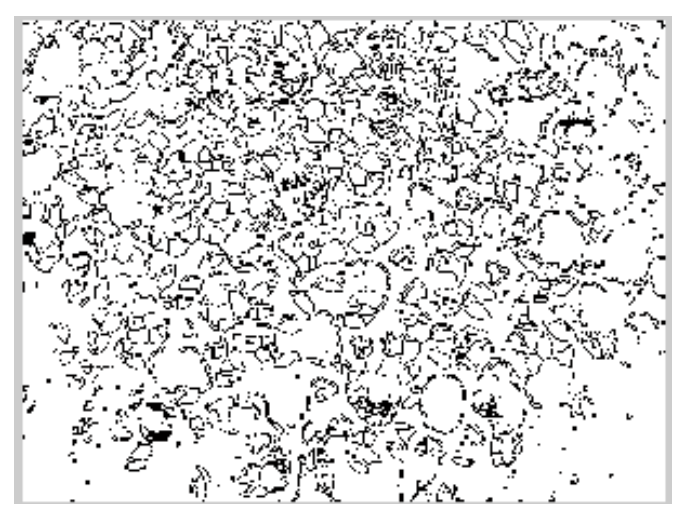

Figure 13: Differences between both segmentation results

As the results shows that the segmented images with the pre processing using random walk has less chances of over or under segmentation. The difference between the segmentation using random walk and without using random walk as a preprocessing step is given. This shows the improvement in the segmentation results. The time complexity of the algor ithm is $\mathrm{O}(10 \mathrm{n})$.

\section{Conclusion and Future work}

This section describes the conclusion of the proposed system and the future plane, which defines how to future expand this research.

The goal of image segmentation process is to identify the segments of the image according to the image characteristic e.g., image color, objects shape etc. The simplified working of the image segmentation system is stated here. The most important step is the image acquisition. Any deficiency during the image acquisition can cause many problems in the result. The image used in this process is taken from image library. The input images are of low contrast. This segmentation process deals with the problem caused by these low contrast images by applying a preprocessing step using random walk. This step enhances the contrast of the input image so that the gradient of the image is strong enough to properly segment the image by using the watershed. After preprocessing step the gradient of the image is finding by converting the input image to grey scale. And this gradient of image is used as the input the image. The results show the improvement in the segmentation results using random walk.

The system will examined only one image at a time. This system can be very helpful for the segmentation of the images which are used in different fields of life. And the image analys is process can be facilitated by this system. The research content of this system was segmentation and image enhancement.

In the paper the 2-D random walk is used for the enhancement of the 2-D images this work can be expanded by using 3-D random walk process on the image.

The work can be expanded to the 3-D images too.

\section{References}

Beucher, S. (1991). The watershed transformation applied to image segmentation. 10th Pfefferkorn Conference on Signal and Image Processing in Microscopy and Microanalysis, 16-19 Sept.

Bieniek, A., \& Moga, A. (2000). An efficient watershed algorith m based on connected components. Pattern Recognition, 33, 907-916. 
Digabel, H., \& Lantuejoul, C. (1977). Iterative algorith ms. In J.-L. Chermant (Ed.), Actes du Second Symposium Europeen d'Analyse Quantitative des Microstructures en Sciences des Materiaux, Biologie et Medecine, Caen, 4-7 October 1977, pp. 85-99. Stuttgart: Riederer Verlag.

Erikson, M. (2005). Two preprocessing techniques based on grey level and geometric thickness to improve segmentation results. Uppsala, Sweden: Centre for Image Analys is, Swed ish University of Agricu ltural Sciences.

Hamarneh, G., \& Li, X. (2009). Watershed segmentation using prior shape and appearance knowledge. Image and Vision Computing, 27(1-2), 59-68.

Hsieh, F-Y., Han, C-C., Wu, N-S., Chuangc, T. C., \& Fana, K-C. (2006). A novel approach to the detection of small objects with low contrast. Signal Processing, 86, 71-83

Hsiesh, F-Y. (2006). A study of watershed transform on image segmentation and data classification. Doctoral Dis sertation, National Central University, Computer science and Information Engineering.

Jung, C. R., \& Scharcanski, J. (2005). Robust watershed segmentation using wavelets. Image and Vision Computing, 23, 661-669.

Li, H., Elmoataz, A., Fadili, J. \& Ruan, S. (2003). An improved image seg mentation approach based on level set and mathe matical morphology. In H. Lu \& T.Zhang (Eds.), Proceedings of the Third International Symposium on Multispectral Image Processing and Pattern Recognition, volume 5286, pp. 851854.

Segmentation (image processing). (n.d.) Retrieved from http://en.wikipedia.org/wiki/Seg mentation (image processing)

\section{Biographies}

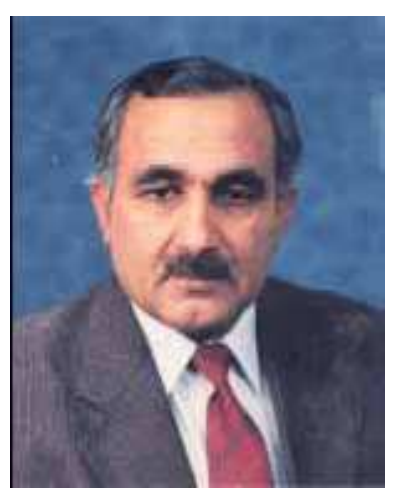

Dr. Malik Sikandar Hayat Khiyal born at Khushab, Pakistan. He is Chairperson Department of Computer Sciences and Software Engineering at Fatima Jinnah Women University, Pakistan. He received his M.Sc degree from Quaid-e-Azam University, Is lamabad. He got first position in the faculty of Natural Science of the University. He was awarded the merit scholarship for Ph.D. He received his Ph.D. degree from UMIST, Manchester, U.K. He developed software of underground flow and advanced fluid dynamic techniques. His areas of interest are Numerical Analysis, Mode ling and Simulation, Discrete structure, Data structure, Analysis of Algorithm, Theory of Automata and Theory of Computation. He has more than fifty research publication published in National and International Journals and Conference proceedings. He can be contacted at m.sikandarhayat@ yahoo.com, Fatima Jinnah Women University, Rawalpindi Pakistan.

Mr. Aihab Khan works in Department of Computer Sciences Fatima Jinnah Women University, Pakistan. His research interests are in the field of Data mining, Data Warehousing as well as Information Security. He can be contacted at aihabkhan@ yahoo.com, Fatima Jinnah Women University, Rawalpindi Pakistan.

Ms. Amna Bibi is a graduate from Department of Computer Sciences, Fatima Jinnah Women University, Pakistan She can be contacted at gul_star3@yahoo.com. 